\title{
Author Correction: PI3K/AKT activation induces PTEN ubiquitination and destabilization accelerating tumourigenesis
}

Min-Sik Lee, Man-Hyung Jeong, Hyun-Woo Lee, Hyun-Ji Han, Aram Ko, Stephen M. Hewitt, Jae-Hoon Kim, Kyung-Hee Chun, Joon-Yong Chung, Cheolju Lee, Hanbyoul Cho \& Jaewhan Song

Correction to: Nature Communications https://doi.org/10.1038/ncomms8769, published online 17 July 2015.

In this Article, there is an error in Fig. 7, in which the PTEN western blot in the IP fraction of Fig. 7c is inadvertently reproduced in the IP fraction of Fig. 7b. This error does not affect the scientific validity of the conclusions, and has not been corrected in the original version of the Article. The correct Fig. 7 is present below. 
a

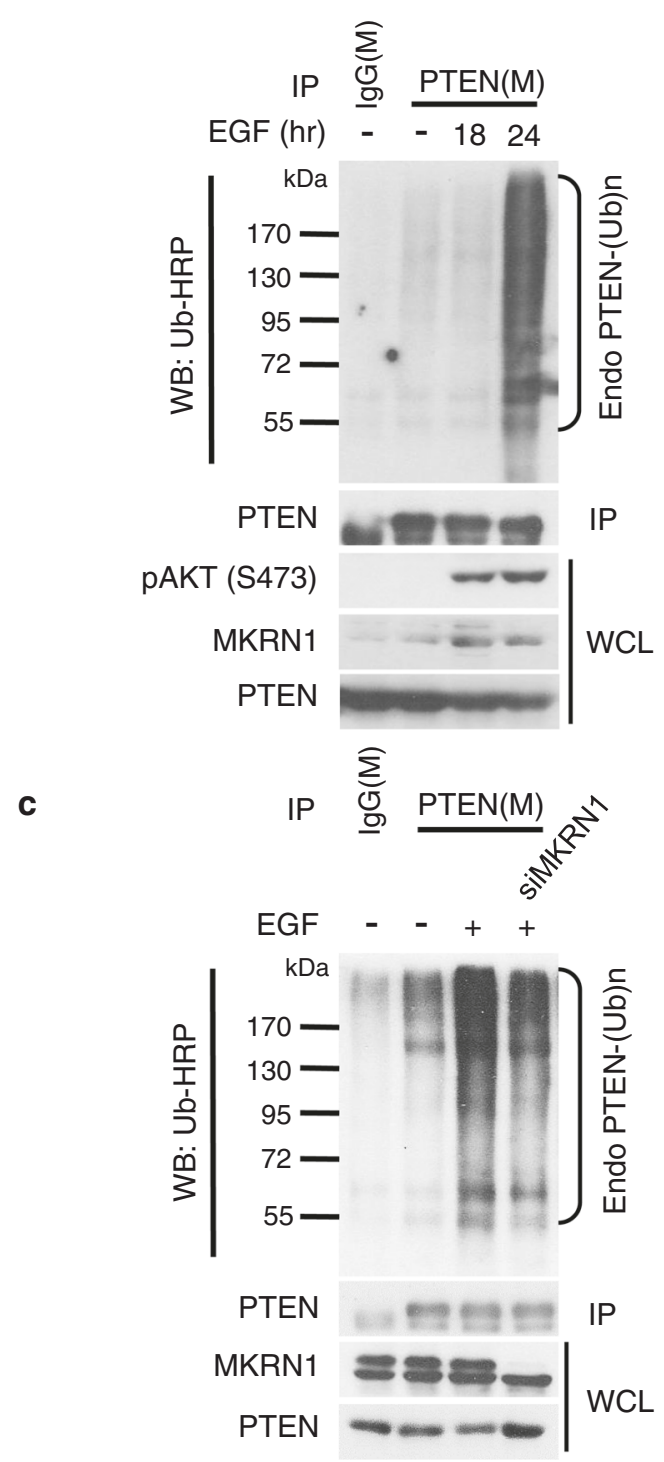

b

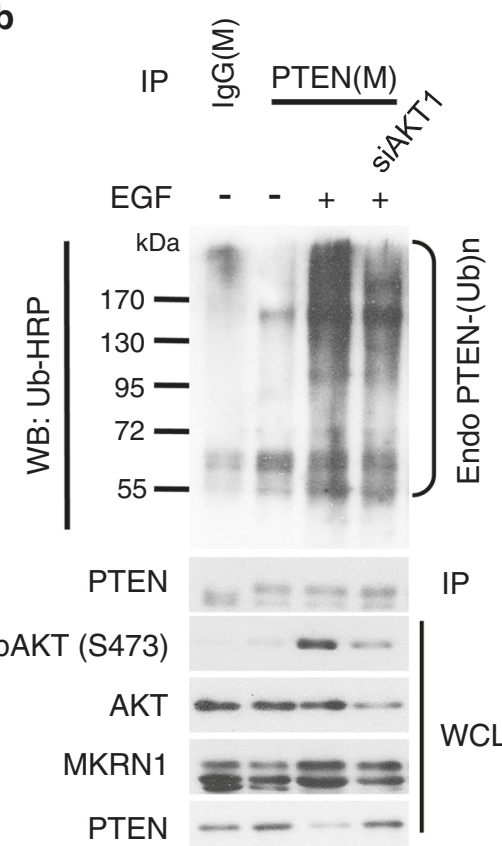

e

d
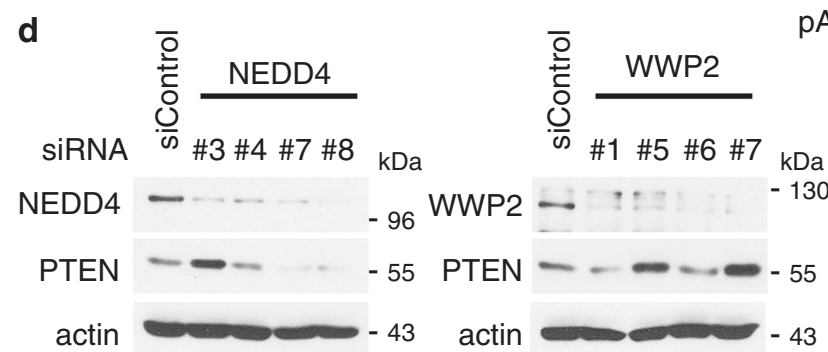
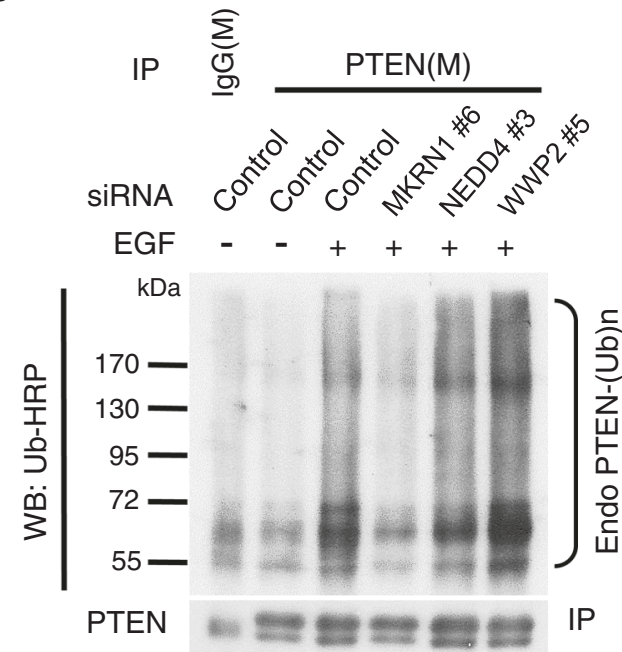

pAKT (S473)

AKT

PTEN

MKRN1

NEDD4

WWP2

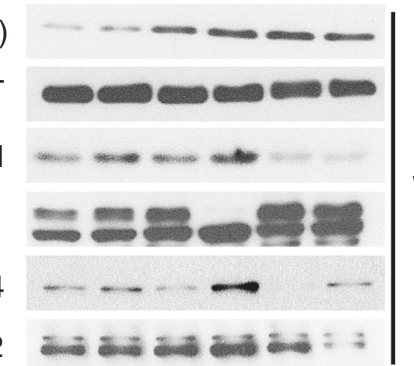

WCL

Fig. 7

Published online: 01 December 2020

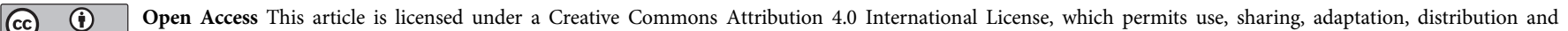
reproduction in any medium or format, as long as you give appropriate credit to the original author(s) and the source, provide a link to the Creative Commons license,

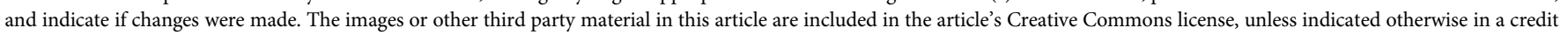

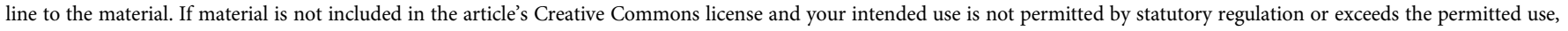
you will need to obtain permission directly from the copyright holder. To view a copy of this license, visit http://creativecommons.org/licenses/by/4.0/. 